\title{
Caracterización del recién nacido pequeño para la edad gestacional. Hospital Leonardo Martínez Valenzuela
}

\author{
Characterization of small for gestational age. Leonardo Martínez Valenzuela Hospital
}

\section{Agueda Ansela Arriola López*}

\section{RESUMEN}

Antecedentes: La Organización Mundial de la Salud (OMS) define a los niños nacidos pequeños para la edad gestacional (PEG) como aquellos cuyo peso al nacer está debajo del percentil 10 por género al nacer, para la edad gestacional. En el 2010, se estimó que 32.4 millones de bebés nacieron PEG en países de medianos y bajos ingresos. Objetivo: Determinar los factores asociados al recién nacido pequeño para la edad gestacional en el Hospital Leonardo Martínez Valenzuela (HLMV) de San Pedro Sula, en el período comprendido de enero a diciembre de 2016. Pacientes y Métodos: Estudio cuantitativo, observacional, descriptivo; se realizó la revisión de 84 expedientes clínicos, se aplicó un cuestionario que constaba de 23 preguntas abiertas y cerradas, evaluando factores de riesgo fetales, maternos y demográficos. Resultados: $55 \%$ de los RN PEG fueron del sexo masculino, 96\% eran RN a término y el $82 \%$ eran de bajo peso al nacer. Se encontraron anomalías fetales como la microcefalia y polidactília $2,3 \%$; los factores maternos encontrados fueron las enfermedades maternas en $9,9 \%$ descritas como la hipertensión gestacional, preeclampsia leve y severa, las infecciones maternas como infección del tracto urinario, vaginosis $y$ fiebre $26,5 \%$, factores demográficos, como la edad entre 18-35 años 83\%, escolaridad baja $89 \%$, ser mestiza, $8 \%$, primigesta $63 \%$, período intergenésico mayor de 2 años $52 \%$. Conclusiones: Es necesario identificar a las gestantes con factores de riesgo como los encontrados para disminuir la condición de pequeños para la edad gestacional.

*Médico Residente tercer año de pediatría.

Dirigir correspondencia a: aaarriola_lopez@hotmail.com

Recibido: 20 de diciembre 2017 Aprobado: 08 de febrero 2018
Se debe actuar poniendo énfasis en los factores modificables.

\section{PALABRAS CLAVE}

Recién nacido, edad gestacional, paridad.

\section{ABSTRACT}

Background: The World Health Organization (WHO) defines children born small for gestational age (SGA) as those whose birth weight is below the 10th percentile by gender at birth, for gestational age. In 2010, it was estimated that 32.4 million babies were born SGAs in low and middle income countries. Objective: To determine the factors associated with the small newborn for gestational age in the Hospital Leonardo Martínez Valenzuela of San Pedro Sula, in the period from January to December 2016. Materials and methods: Quantitative, observational, descriptive study; a review of 84 clinical files was carried out, a questionnaire was applied consisting of 23 open and closed questions, evaluating fetal, maternal and demographic risk factors. Results: $55 \%$ of the SGA newborns were male, $96 \%$ were full term and $82 \%$ were low birth weight. Fetal abnormalities such as microcephaly and polydactyly $2.3 \%$ were found; the maternal factors found were maternal diseases in $9.9 \%$ described as gestational hypertension, mild and severe preeclampsia, maternal infections such as urinary tract infection, vaginosis and fever $26.5 \%$, demographic factors, such as age between 18- 35 years $83 \%$, low schooling $89 \%$, being mestizo, $8 \%$, primiparous 63\%, intergenesic period greater than 2 years $52 \%$. Conclusions: It is necessary to identify pregnant women with risk factors such as those found to reduce the condition of small for gestational age. We must act with an emphasison modifiable factors. 


\section{KEY WORDS}

Newborn, gestational age, parity.

\section{INTRODUCCIÓN}

La OMS define a los niños nacidos PEG como aquéllos cuyo peso al nacer está por debajo del percentil 10 por género al nacer, para la edad gestacional. ${ }^{(1,2,3)}$ En el 2010, se estima que 32.4 millones de bebés han nacido pequeños para la edad gestacional en países de medianos y bajos ingresos.

Las tasas más altas se encontraban en el sur de Asia. ${ }^{(4)}$ En Latinoamérica, una evaluación realizada entre 1999 y 2004 en Colombia, reveló que el $3,6 \%$ de 14,274 eran recién nacidos pequeños para la edad gestacional. En México, una revisión de 31,209 niños nacidos entre el año 2000 y 2002 mostró una prevalencia de PEG del $6 \%$ en la población general. ${ }^{(2)}$ En el año de 1995 se realizó en Honduras un estudio analítico, longitudinal y prospectivo en el que se estimó que el $11 \%$ de los recién nacidos nacen con bajo peso al nacer y que el $20 \%$ de los recién nacidos que mueren en nuestro país son RNBPN.(5) En 1996 una investigación retrospectiva y descriptiva de 210 fallecimientos en el Servicio de Neonatología de la Unidad Materno Infantil Instituto Hondureño de Seguridad Social de Tegucigalpa, Honduras, encontrándose una tasa de mortalidad neonatal de 9.3 por cada mil nacidos vivos, la cual incrementa a 15.2 entre los de bajo peso al nacer. ${ }^{(6)}$

Considerando las altas tasas de morbilidad y mortalidad en esta población, ${ }^{(7)}$ es primordial su rápida identificación. Los RN PEG tienen 5 veces más probabilidad de morir en el período neonatal y 4,7 veces más probabilidad de morir en su primer año de vida. El nacimiento prematuro y el bajo peso al nacer son causas importantes de muerte en los países de bajos y medianos ingresos. ${ }^{(8)}$

Los RN PEG tienen mayor riesgo de desarrollar hipertensión y diabetes tipo 2 en la edad adulta. También presentan mayor riesgo de tener talla baja, alteraciones en su neurodesarrollo(9) y menor coeficiente intelectual (Cl). Los niños de bajo peso al nacer con "catch up" del crecimiento temprano, tienen mayor riesgo de desarrollar obesidad en la infancia y enfermedades del adulto, incluyendo enfermedad coronaria, accidente cerebro vascular y diabetes mellitus, las cuales, según la OMS, se encuentran entre las 10 causas principales de muerte a nivel mundial. ${ }^{(10)}$ Es imperativo conocer las características de este grupo de recién nacidos con la finalidad de identificarlos de forma oportuna y crear estratégias eficaces de intervención.

\section{PACIENTES Y MÉTODOS}

Se realizó un estudio cuantitativo, observacional y descriptivo en el HLMV de San Pedro Sula, Honduras. Se identificaron los recién nacidos pequeños para su edad gestacional; los que se encuentran por debajo del percentil 10 para su peso, según edad y sexo, nacidos en el período de enero a diciembre del 2016 de los listados de Labor y Parto, se clasificaron de acuerdo a las curvas de Jurado-García, se obtuvieron los datos de los expedientes clínicos en el área de estadística de dicho centro hospitalario.

El criterio de inclusión fue: todo expediente clínico y hoja perinatal de recién nacido $P E G$ que se encontraron completos en el área de estadística del Hospital y se excluyeron los expedientes clínicos incompletos, se llenó un formulario que constaba de 23 preguntas abiertas y cerradas, el cual fue validado previamente mediante una prueba piloto.

El universo lo conformaron todos los recién nacidos PEG nacidos en el área de Labor y Parto del HLMV en el período de enero a diciembre de 2016. El tamaño de la muestra fue de 84 pacientes. El método de muestreo fue probabilístico por conveniencia.

La información fue ingresada en una base de 
datos (Programa Epi Info 3.5 para Windows 3.5, CDC, Atlanta, GA, EUA). Los resultados se presentaron como frecuencias y porcentajes de las variables estudiadas.

\section{RESULTADOS}

El universo lo conformaron 631 recién nacidos PEG nacidos de enero a diciembre del 2016, el tamaño de la muestra fue de 84 pacientes para un nivel de confianza de $95 \%$. El $55 \%(n=46)$ de los RN PEG son del sexo masculino. El $96 \%(n=81)$ son a término, 4\% $(n=3)$ son recién nacidos pretérmino, no se encontraron recién nacidos post término (ver gráfico No. 1). El 82\% ( $n=69)$ de los RN PEG presentaron bajo peso al nacer, es decir peso $<2500$ gramos. El 81\% $(n=68)$ de los RN PEG se enviaron a alojamiento conjunto con la madre, el $15,5 \%(n=13)$ de los mismos preciso de ingreso al área de neonato del hospital y $2,4 \%(n=2)$ fue referido a otro hospital (Hospital Nacional Mario Catarino Rivas).

Gráfico No. 1: Edad gestacional de los recién nacidos pequeños para la edad gestacional.

\section{Edad gestacional}

- Prematuros moderados a tardíos $\mathrm{A}$ término

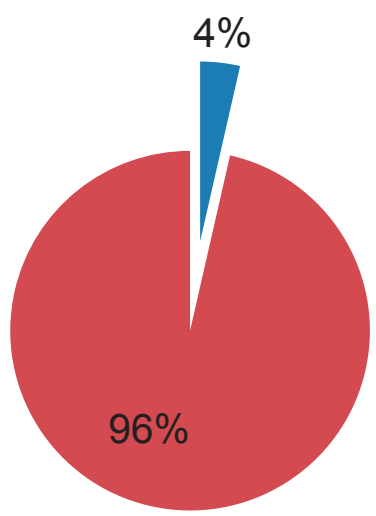

Fuente: Instrumento de recolección de datos

Los RN PEG hospitalizados fueron un total de $(n=13)$ de estos el $32 \%(n=6)$ fueron ingresados bajo el diagnóstico de taquipnea transitoria del recién nacido, $11 \%$ por síndrome de aspiración meconial, $11 \%$ riesgo de sepsis por fiebre materna, $11 \%$ infección del tracto urinario materno, $11 \%$ restricción del crecimiento intrauterino, 5\% leucocitosis, 5\% hijo de madre con chikungunya, 5\% neumonía connatal, 5\% riesgo alto de sepsis por parto extra hospitalario, 5\% potencialmente séptico por manipulación vía aérea.

Los diagnósticos de los RN PEG que requirieron referencia fueron: neumonía connatal y taquipnea transitoria del recién nacido.

Los factores fetales encontrados en los RN PEG fueron anomalías congénitas como: microcefala $1 \% \quad(n=1)$ y polidactília cubital $1 \%(n=1)$, además de infecciones como neumonia connatal $1 \%$. $(n=1)$. El $83,3 \%(n=70)$ de las madres se encuentran en el grupo de edad comprendido entre los 18-35 años, el $15,5 \%(n=13)$ eran menores de 18 años y el $1,2 \%(n=1)$ son mayores de 35 años (Ver gráfico No. 2). El 62\% ( $n=52)$ de las madres son procedentes del municipio de San Pedro Sula. El $95,2 \%(n=80)$ de las madres eran mestizas. El $42,8 \%(n=36)$ de las madres curso primaria completa, el 1,1\% $(n=1)$ de las mismas alcanzó la educación superior.

Gráfico No. 2: Edad de las madres de los recién nacidos pequeños para su edad gestacional.

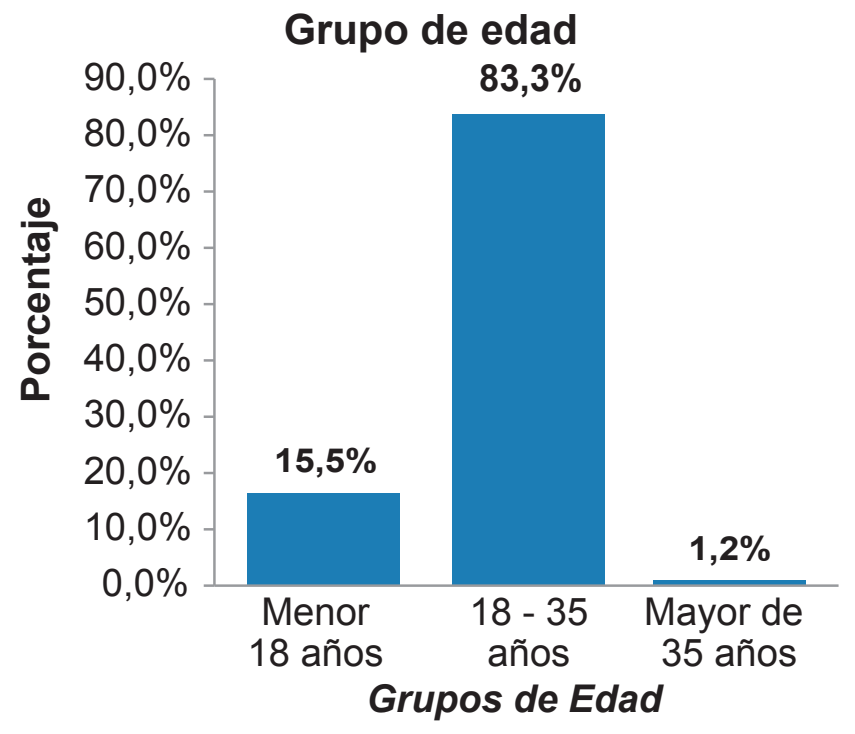

Fuente: Instrumento de recolección de datos 
El $61 \%(n=51)$ de las madres eran ama de casa, el $20 \%(n=4)$ corresponden a enfermedades médicas, hipertensión gestacional, preeclampsia leve y severa. El 40\% (n=8) de ellas tenían infecciones asociadas, siendo las infecciones del tracto urinario las más frecuentes en un 25\%, $(n=5)$; de las madres con peso y talla consignados del $10 \%(n=2)$ tenían bajo peso. (Ver Tabla No. 1).
El $63 \%(n=53)$ de las madres eran primigestas, el $26 \%(n=22)$ secundigestas, el $10 \%$ $(n=8)$ multíparas y el $1 \%(n=1)$ gran multípara. El 52\% $(n=16)$ de las madres tenía un período intergenésico mayor de 2 años y el $48 \%(n=15)$ menor de 2 años.

Tabla No. 1: Factores maternos asociados a los recién nacidos pequeños para la edad gestacional.

\begin{tabular}{|c|c|c|c|}
\hline Categoría & Detalle & Frecuencia & Porcentaje \\
\hline \multirow{5}{*}{ Enfermedades Médicas } & Preclampsia leve & 1 & $1 \%$ \\
\hline & Hipertensión gestacional & & \\
\hline & Chikungunya & 1 & $1 \%$ \\
\hline & Preclampsia severa & 1 & $1 \%$ \\
\hline & Asma Bronquial & 1 & $1 \%$ \\
\hline \multirow[t]{3}{*}{ Infecciones de la Madre } & Fiebre & 1 & $1 \%$ \\
\hline & Infección del tracto urinario & 5 & $6 \%$ \\
\hline & Vaginosis & 2 & $2 \%$ \\
\hline Uso de Fármacos & No consignado & 0 & $0 \%$ \\
\hline \multirow[t]{5}{*}{ Estado nutricional } & Bajo peso & 2 & $2 \%$ \\
\hline & Peso normal & 10 & $12 \%$ \\
\hline & Obesidad & 1 & $1 \%$ \\
\hline & Sobrepeso & 3 & $4 \%$ \\
\hline & No consignado & 68 & $81 \%$ \\
\hline
\end{tabular}

Fuente: Instrumento de recolección de datos

\section{DISCUSIÓN}

En el estudio el 55\% de los RN PEG correspondían al sexo masculino, concordando con lo encontrado en el estudio de García-Basteiro et al, realizado en el Sur de Mozambique en el período 2010-2012 donde se encontró que el $51,4 \%$ de los $\mathrm{RN}$ nacidos PEG correspondían al sexo masculino. ${ }^{(7)}$

Del total de RN PEG de nuestro estudio el $96 \%(n=81)$ corresponden a RN nacidos a término y el $4 \%(n=3)$, discrepando con lo encontrado en la literatura donde en el estudio de Black R. sobre prevalencia de los recién nacidos pequeños para su edad gestacional a nivel mundial, publicado en el
2015, estima que, hasta el $20 \%$ de los RN PEG nacidos en países del sur de Asia y algunos países de África, son pretérmino. ${ }^{(1)}$

El 82\% (n=69) de los RN PEG tenían bajo peso al nacer, es decir un peso inferior a 2500 gramos, mostrando una relación directa entre ser PEG y tener bajo peso al nacer, encontrando en nuestro estudio incluso cifras mayores a las descritas en otros artículos, ya que en países asiáticos se han encontrado prevalencias de hasta $10,3 \%{ }^{(1,7)}$ no encontramos en la literatura una relación entre ser PEG y la talla, pero en nuestro estudio estimamos que el $82 \%(n=69)$ de nuestros RN PEG presentaron talla inferior a 48 centímetros. 
El $15,5 \%(n=13)$ de los RN PEG requirieron de hospitalización, siendo la taquipnea transitoria del recién nacido en un $32 \%(n=6)$, el principal diagnóstico de ingreso. El $81 \%$ $(n=68)$ luego de su nacimiento y de recibir los cuidados inmediatos del recién nacido, se alojaron conjuntamente con la madre, indicando que a pesar de ser PEG, no presentaron mayor morbilidad, coincidiendo con otros estudios, como el estudio Marzouk et al, quienes en el 2011 estimaron los costos que generan a nivel nacional, de los hospitales franceses, ser PEG, encontrando que el $10,9 \%$ requirió de hospitalización a lo largo de su primer año de vida. ${ }^{(3)}$

De acuerdo a la OMS, Las anomalías congénitas afectan a uno de cada 33 neonatos y causan 3,2 millones de discapacidades al año. Se calcula que $2-5 \%$ de los $R N$ vivos y $10 \%$ de los RN muertos, presentan algún defecto congénito. ${ }^{(11)}$ En nuestro estudio el $4 \%$ de los RN PEG presentó algún tipo de anomalía congénita; microcefalia y polidactília cubital; el $96 \%$ no presentó ningún factor de riesgo fetal.

Kozuki et al, en 2013, analizaron datos de 14 estudios de cohorte donde encontraron que las madres menores de 18 años tienen mayor riesgo de tener un RN PEG. Una explicación plausible puede ser, el incompleto crecimiento biológico de la madre y a una desnutrición relativa, que está relacionado con la edad ginecológica de la madre más que con la edad cronológica; diferenciándose de los resultados obtenidos en este estudio donde se encontró que las madres en la edad comprendida entre 18 y 35 años se asocian más a los RN PEG, en un $83 \%$ $(\mathrm{n}=70)^{(12)}$

El $62 \%(n=52)$ de las madres de los $R N$ PEG eran procedentes del Municipio de San Pedro Sula, el 95\% ( $n=80)$ eran mestizas, en vista de la ausencia de estudios locales similares, estos resultados no pueden ser corre- lacionados con otra literatura. $61 \%(n=51)$ de las madres eran amas de casas, no se encontró en la literatura asociación con la ocupación materna.

El $10 \%(n=4)$ de las madres presentaron enfermedades médicas tipo hipertensión gestacional, preeclampsia leve y severa, ${ }^{(13)}$ que se asociaron con el nacimiento de RN $P E G$, relación que está ampliamente descrita en otros estudios ${ }^{(1,7,14)}$ El estudio de Cohorte retrospectiva de Mariaca et al, que utilizó datos del Sistema Informático Materno Perinatal del Hospital María Auxiliadora de Lima, del período 2000 a 2010, con ( $n=64$ 670) gestantes, encontró que el haber presentado preeclampsia duplicó el riesgo para el recién nacido PEG y la eclampsia lo triplicó. Se sustenta que el mecanismo fisiopatológico implicado es una disrupción de la vasculatura úteroplacentaria y/o un volumen sanguíneo materno reducido a nivel placentario. ${ }^{(15)}$

El $10 \%(n=8)$ de las madres de este estudio documentaron infecciones durante el embarazo, predominando las infecciones del tracto urinario en un $62 \%(n=5)$ vaginosis, $25 \%$ $(n=2)$, discrepando con la literatura, que reporta que el nacimiento de RN PEG se han asociado a infecciones maternas tales como Rubeola, Citomegalovirus, VIH, Herpes virus, Tripanosomiasis, según Paisan et al. ${ }^{(16)}$

El tabaquismo materno constituye uno de los factores ambientales más importantes para prevenir ser PEG, esto ocurre tanto por la vasoconstricción placentaria inducida por la nicotina como por la hipoxia crónica por el aumento de carboxihemoglobina.(10) En nuestro estudio ninguno de los expedientes clínicos consignaba el consumo de tabaco, drogas ilícitas y fármacos, lo cual constituye una limitante para asociar a los RN PEG del Hospital Leonardo Martínez Valenzuela con dicho factor de riesgo. 
Otro factor de riesgo asociado al PEG es el estado nutricional materno, específicamente el bajo peso previo al embarazo y durante el mismo, se ha observado que una disminución en el consumo de alimentos por la madre o de su capacidad de absorción, puede causar menor crecimiento fetal además de defectos persistentes en el producto, el impacto a largo plazo dependerá del estadío en el que haya mala nutrición, de su duración e intensidad, ${ }^{(17)}$ Tejeda-Maria- ca et al, en 2015, determinó que el bajo peso materno y ser primigesta son factores de riesgo. ${ }^{(7)}$ En nuestro estudio el $81 \% \quad(n=68)$ de las madres no tenían su peso consignado en la historia perinatal, restringiendo la asociación entre el estado nutricional materno y el nacer PEG y el $53 \% \quad(n=63)$ de las madres eran primigestas. ${ }^{(18)}$
En el estudio de cohorte, realizado en 2002 en Complejo Hospitalario Metropolitano de la Caja del Seguro Social de Panamá, determinaron que un período intergenésico menor de 6 meses constituye un factor de riesgo para nacer pretérmino y PEG, además de que un período intergenésico mayor de 49 meses predispone a Preeclampsia, en nuestro estudio el $48 \%(n=16)$ presentó período intergenésico corto. ${ }^{(19)}$ No es posible asociar el tener un hijo previo para la edad gestacional ya que este dato no está consignado en los expedientes clínicos, debido a que este no es parte de la historia perinatal.

\section{REFERENCIAS BIBLIOGRÁFICAS}

1. Black R. Global Prevalence of Small for Gestational Age Births. NestleÂ Nutr Inst Work Ser. 81:1-7. 2015.

2. Boguszewski M, Mericq V, Bergada I, Damiani D, Belgorosky A, Gunczle P et al. Consenso Latinoamericano: niños pequeños para la edad gestacional. Revista Chilena de Pediatría. 83 (6): 620634. 2012.

3. Marzouk A, Filipovic-Pierucci A, Baud O, Tsatsaris V, Ego A, Charles MA et al. Prenatal and post-natal cost of small for gestational age infants: a national study. BMC Health Services Research. 17:221. 2017.

4. Lee A, Katz J, Blencowe H, Cousens S, Kozuki N, Vogel $\mathrm{J}$ et al. National and regional estimates of term and preterm babies born small for gestational age in 138 low-income and middle-income countries in 2010. Lancet Glob Health. 1:e26-36. 2013.

5. Reyes D, Fernández-Zelaya K, Osorio-Murillo $A$, Zelaya-Lozano E, Pineda-Barahona E, Dala-Sierra E. Curvas De Crecimiento De Niños Hondureños De Bajo Peso Al Nacer $Y$ Normales Durante El Primer Año De Vida. Rev Med Post UNAH. 1(1): 2-5. 1996.

6. Banegas O, Pineda-Barahona E, Meiía N. Mortalidad Neonatal En Niños De Bajo Peso Al Nacer En El Servicio De Neonatología Del I.H.S.S. Rev med Post UNAH. 3(1): 78-84. 1998.

7. Garcia-Basteiro A, Quinto L, Macete E, Bardajõ A, Gonzalez R, Nhacolo A, et al. Infant mortality and morbidity associated with preterm and small-for-gestational-age births in Southern Mozambique: A retrospective cohort study. 12(2): 1-14. 2017. 
8. Kc A, Wrammert J, Nelin V, Ewald U, Clark R, Malqvist M. Level of mortality risk for babies born preterm or with a small weight for gestation in a tertiary hospital of Nepal. BMC Public Health. BMC Public Health.15: 877. 2015.

9. Mello B, Gagliardo H, Gonçalves V Neurodevelopment of small-for-gestational age infants: behavioral aspects in first year Arq Neuropsiquiatr. 72(7): 517-523. 2014.

10. Biosca Pàmiesa M, Rodríguez Martíneza G, Samper Villagrasaa M, Odriozola Grijalbac M, Cuadrón Andrésa L, et al. Aspectos perinatales, crecimiento y tipo de lactancia de los nacidos pequeños para su edad gestacional. An Pediatr (Barc). 78(1): 14-20.2013.

11. Mazzi E. Defectos congénitos. Rev. bol. ped. 54 (3). 2015.

12. Kozuki N, Lee A, Mariangela F Silveira3, Ayesha Sania4, Joshua P Vogel5,6, Linda Adair7.The associations of parity and maternal age with small-for-gestational-age, preterm, and neonatal and infant mortality: a meta-analysis. BMC Public Health.13(3):S2.2013.

13. Collins S, Welsh A, Impey L, Noble A, Stevenson G. 3D fractional moving blood volume (3D-FMBV) demonstrates decreased first trimester placental vas cularity in pre-eclampsia but not the term, small for gestation age baby. PLOS. 2017.
14. Zerquera-Rodriguez J, Cabada-Martínez Y, Zerquera-Rodríguez D, María- Delgando-Acosta $\mathrm{H}$. Factores de riesgo relacioados con bajo peso al nacer en el municipio Cienfuegos. Medisur [revista en Internet]. 2015 [citado2015Jun20 ]; 13 (3): [aprox.8p.].

Disponible en: http://www.medisur.sld. cu/index.php/medisur/article/view/2861.

15. Tejeda-Mariaca J, Pizango-Mallqui $\mathrm{O}$, Alburquerque-Duglio M, Mayta-Tristán P. Factores De Riesgo Para El Neonato Pequeño Para La Edad Gestacional En Un Hospital De Lima, Perú Rev Peru Med Exp Salud Publica.32(3): 449-56. 2015.

16. Paisán Grisolía L, Sota Busselo I, Muga Zurriarían O, Imaz Murgiondo M. El recién nacido de bajo peso. Protocolos Diagnóstico Terapeúticos de la AEP: Neonatología. 78-84.2008.

17. Perea-Martínez A, López-Navarrete G, Carbajal-Rodríguez L, Rodríguez-Herrera $\mathrm{R}$, Zarco-Román J, Loredo-Abdalá A. Alteraciones en la nutrición fetal y en las etapas tempranas de la vida. Su repercusión sobre la salud en edades posteriores. Acta Pediatr Mex.33(1): 26-31. 2012.

18. Tejeda-Mariaca J, Pizango-Mallqui O, Alburquerque-Duglio $\mathrm{M}$, Mayta-Tristán P. Factores De Riesgo Para El Neonato Pequeño Para La Edad Gestacional En Un Hospital De Lima, Perú Rev Peru Med Exp Salud Publica.32(3): 449-56 2015.

19. Domínguez L, Vigil-De Gracia P. El intervalo intergenésico: un factor de riesgo para complicaciones obstétricas y neonatales. Clin Invest Gin Obst. 32(3): 122-6. 2005. 